06.01 - Epidemiology

\title{
11947
}

\section{Respiratory disease phenotypes in a general population sample: latent transition analysis}

Epidemiology, Asthma, COPD

\author{
S. Maio ${ }^{1}$, S. Fasola ${ }^{2}$, S. Baldacci ${ }^{1}$, L. Carrozzi ${ }^{3}$, F. Pistelli ${ }^{4}$, M. Simoni ${ }^{1}$, S. La Grutta ${ }^{2}$, V. \\ Muggeo $^{5}$, G. Viegi ${ }^{2}$
}

${ }^{1}$ Pulmonary Environmental Epidemiology Unit, CNR Institute of Clinical Physiology - Pisa (Italy), ${ }^{2}$ CNR Institute of Biomedicine and Molecular Immunology (IBIM) "A. Monroy" - Palermo (Italy), ${ }^{3}$ Department of Surgical, Medical, Molecular Pathology and Critical Area, University of Pisa and Pulmonary Unit, University Hospital of Pisa - Pisa (Italy), ${ }^{4}$ Unit of Pulmonology, Cardio-Thoracic and Vascular Department, University Hospital of Pisa - Pisa (Italy), ${ }^{5}$ Dept of Economics, Business and Statistics, Palermo University - Palermo (Italy)

Background: Few data are available on the temporal pattern of respiratory disease phenotypes in general population.

Aim: To detect longitudinal patterns of disease phenotypes related to risk factors and physician visits.

Methods: Pisan general population sample from 2 cross-sectional studies (PI1: 1991-93; PI2: 2009-11; $\mathrm{n}=1107$ ), questionnaire-based data. Latent transition analysis (LTA) was performed to assess respiratory disease phenotypes at PI1 and PI2, labelled according to disease/symptom occurrence. Possible patterns were persistence, worsening and improvement of the phenotype. Multiple logistic regression models were estimated to assess the association among phenotype patterns, risk factors (smoking habits-SH and occupational exposure-OE) and physician visits, adjusting for age, sex and educational level.

Results: 4 cross-sectional phenotypes were ranked by severity in PI1 and in PI2: healthy $(H)(59$ and $55 \%)$, allergic rhinitis (AR) (28 and 27\%), cough/phlegm (CP) (11 and 15\%), asthma (A) (3 and $4 \%$ ). The longitudinal patterns were: persistent $\mathrm{H}(53 \%)$, persistent AR $(27 \%)$, persistent CP $(9 \%)$, improvement $(2 \%)$, worsening $(10 \%)$.

Significant associations: persistent CP with persistent $\mathrm{SH}$ (OR 5.9), persistent OE (OR 2.9) and incident $\mathrm{OE}$ (OR 2.2); persistent $\mathrm{CP}$ and worsening of phenotype with current visits of family physician (OR 5.4 and OR 4.7 , respectively) and of specialist (OR 2.7 and OR 3.9 , respectively).

Conclusions: LTA allowed to identify four different phenotypes based on respiratory symptoms/diseases and their longitudinal patterns over 18 years. Such analysis brings new perspectives in the analyses of population-based data. 
